Journal of Advanced Research in Fluid Mechanics and Thermal Sciences

\title{
Dual Solutions in Mixed Convection Flow Along Non-Isothermal Inclined Cylinder Containing Gyrotactic Microorganism
}

\author{
Nayema Islam Nima ${ }^{1,2}$, Mohammad Ferdows ${ }^{1, *}$ \\ Research group of Fluid Flow Modelling and Simulation, Department of Applied Mathematics, University of Dhaka, Dhaka, Bangladesh \\ Department of Quantitative Sciences, International University of Business Agriculture and Technology, Dhaka, Bangladesh
}

\section{ARTICLE INFO ABSTRACT}

Article history:

Received 9 May 2021

Received in revised form 15 August 2021

Accepted 19 August 2021

Available online 29 September 2021

\section{Keywords:}

Mixed convection; Inclined Cylinder; Non-Isothermal; Gyrotactic Microorganism; Dual Solution

\begin{abstract}
The purpose of this research is to present dual solution for combined free and forced convection flow towards a non-isothermal permeable inclined cylinder containing gyrotactic microorganism. Though several researches were done on dual solutions for mixed convection and also along the vertical cylinder for the numerous engineering applications but very few works have done on dual solutions for mixed convection with gyrotactic microorganisms. Two steps are performed here to carry out numerical calculations. Firstly, the governing partial differential equations are simplified into set of coupled non-linear ordinary differential equations using similarity transformations and then solved numerically using bvp4c function from MATLAB. Dual solutions are observed for heat, mass and density of motile microorganism transfer rate and also for velocity, temperature, concentration, and microorganism profile beyond a critical point. The research is reached to excellent argument by comparison in few cases between the results obtained from MATLAB and Maple algorithm. The heat, mass and motile microorganism transfer rate decreases from free to mixed convection regime and then increases to forced convection regime with the influence of different flow control parameters. The results also indicate that dual solutions for different flow profiles exist only in free convection dominated regime.
\end{abstract}

\section{Introduction}

In the last few decades, the combined (free and forced) or mixed convection problems have attracted a great deal of attention from researchers because of its wide range of applications in scientific fields such as engineering, physics, biology, fluid mechanics, and also industrial applications like heat exchanges placed in a low velocity environment, solar collectors exposed to wind currents, atmospheric boundary layer flows, nuclear reactors cooled during emergency shutdown, and various electronic equipment. Mixed convection flow occurs when forced and free convection structures substantially contribute to heat transfer. Several studies were done on mixed convection flow through porous media by many researchers [1-3]. Very recently mixed convection through porous media with heat generation is studied in several researches [4-6]. Gangadhar et al., [7] studied mixed

\footnotetext{
* Corresponding author.

E-mail address: ferdows@du.ac.bd

https://doi.org/10.37934/arfmts.87.3.5163
} 
convection boundary layer flow for casson fluid, Zhao [8] investigated the convection for viscoelastic fluid and Bakar and Roslan [9] studied mixed convection in Lid-Driven Cavity in the presence of Heat Generation.

Flow over vertical cylinder has become great interest to the authors because of its wide range of applications in insulation of vertical porous pipes, connection with oil/gas lines, underground electrical power transmission lines, and disposal of radioactive waste, polymer process, heating or cool of sheets/films. Popiel [10], Loganathan and Eswari [11] observed natural convection flow for cylindrical surfaces. Sewucipto and Yuwono [12] observed influence of upstream installation for cylindrical surface. Rashidi et al., [13] and Dhanai et al., [14] studied combined free and forced or mixed convective nanofluid flow over inclined surfaces and very recently Rihan [15] and Mkhatshwa et al., [16] studied mixed convection flow over vertical cylinder. Convection with power law wall temperature distribution were studied by Ali [17] and Ferdows et al., [18] where Ferdows et al., [18] investigated wall temperature variations for natural convection along vertical plate and Muñoz-Cobo et al., [19], Kim et al., [20] investigated for cylindrical surfaces.

The study of microorganism has become greater attention of researchers to observe the development of microorganisms in bioconvection. Major applications of bioconvection phenomena are noticed in many Microsystems and some noticeable applications in the fields of biomedicine (nanodrug delivery, cancer therapeutics), bio-Microsystems (enzyme biosensors, biotechnology). Bioconvection can be categorized as development process in the field of fluid flow, which acts with the steps of self-propelled up swimming microorganisms such as algae and bacteria that contain oxytaxis, gyrotaxis or gravitaxis organisms. As motile microorganisms are denser than their surrounded liquid and generally swim in the upward direction which produces different flow profiles into the system. The benefits of adding motile microorganisms to the suspension include enhanced mass transfer, microscale mixing, and anticipated improved stability of the fluid. The stability and development of gyrotactic microorganism in a depth cavity were observed by Gorai and Hill [21-22]. Mixed convection nanofluid flow containing gyrotactic microorganisms is observed by several researchers [23-26]. In recent works, Sudhagar et al., [27], Mallikarjuna et al., [28], and Rashad and Nabwey [29], explored mixed convection nanofluid flow over vertical circular cylinder containing gyrotactic microorganisms, Mahdy and Nabwey [30] studied time-mixed convection nanofluid flow for rotating sphere containing motile microorganisms.

In convective heat transfer for solving highly complex non-linear problems sometimes we get multiple (dual) solutions. In many cases it is important to compute unstable states along with stable one, since unstable solutions often interact with stable solutions which produce indescribable phenomena. The study on the presence of dual solutions in mixed convective boundary layer flows may bring new vision on engineering applications [31]. Probably, Ridha and Curie [32] was the first who found existence of dual solution for opposing flow at first. After that Subhashini et al., [33] extended that research for assisting flow. Dual solutions for mixed convection boundary layer flow were firstly investigated by Ingham [34] and also Merkin [35]. Rostami et al., [36] and Salleh et al., [37] studied mixed convection in the presence nanofluid. Very recently Khan et al., [38] also presented dual solution for mixed convection with silica-alumina hybrid nanofluid for curved surface.

The objective of this article is to present dual solution for combined free and forced convection over inclined cylinder containing gyrotactic microorganism. Though Rostami et al., [36], Salleh et al., [37], Khan et al., [39], and Shu et al., [40] investigated dual solutions for mixed convection and also along the vertical cylinder but according to the authors' knowledge very few works by Sudhagar et al., [27] and Ferdows et al., [41] have done on dual solutions for mixed convection with gyrotactic microorganisms [39-40]. So, the presented results of dual solution in case of mixed convection over inclined cylinder are entirely unique. 


\section{Methodology}

\subsection{Mathematical Formulation}

Consider the steady free forced convection boundary layer flow over an inclined cylinder of radius $r_{0}$ implanted in a saturated permeable medium containing gyrotactic microorganism as shown in Figure 1 . It is assumed that the mainstream velocity $U(x)$ and cylinder surface is maintained in constant temperature $T_{w}$, Concentration of fluid $C_{w}$ and motile microorganism concentration $n_{w}$, while far from the surface of cylinder the velocity, temperature and concentrations are $u_{\infty}, T_{\infty}, C_{\infty}$, and $n_{\infty}$. The axial and radial coordinates are considered to be $\mathrm{x}$ and $\mathrm{r}$ where $\mathrm{x}$-axis measured vertically upward along the axis of the cylinder and $r$-axis measured normal to $x$-axis. The gravitational acceleration $g$ acts in the downward direction in opposition to the $x$-direction.

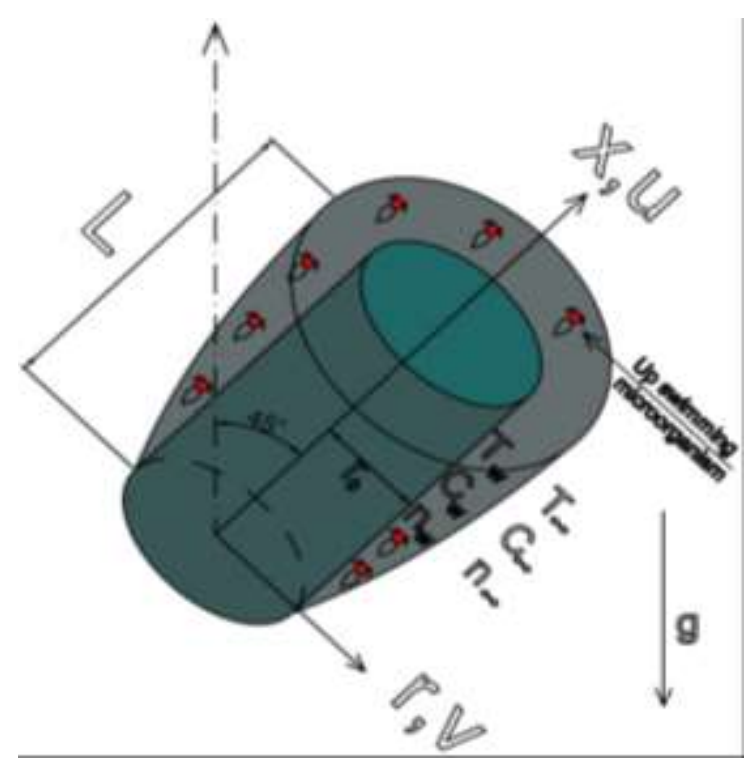

Fig. 1. Physical model and Coordinate system

Under the assumptions along with the physical phenomena and Boussinesq approximations, the governing equations are:

$$
\begin{aligned}
& \frac{\partial(r u)}{\partial x}+\frac{\partial(r v)}{\partial r}=0 \\
& \frac{\partial u}{\partial r}= \pm \frac{g K}{v}\left(\beta_{T} \frac{\partial T}{\partial r}+\beta_{C} \frac{\partial C}{\partial r}+\beta_{n} \frac{\partial n}{\partial r}\right) \cos \omega \\
& u \frac{\partial T}{\partial x}+v \frac{\partial T}{\partial r}=\alpha\left(\frac{1}{r} \frac{\partial}{\partial r}\left(r \frac{\partial T}{\partial r}\right)\right) \\
& u \frac{\partial C}{\partial x}+v \frac{\partial C}{\partial r}=D_{m}\left(\frac{1}{r} \frac{\partial}{\partial r}\left(r \frac{\partial C}{\partial r}\right)\right) \\
& u \frac{\partial n}{\partial x}+v \frac{\partial n}{\partial r}+\frac{b W_{c}}{\nabla C}\left(\frac{\partial}{\partial r}\left(n \frac{\partial C}{\partial r}\right)\right)=D_{n}\left(\frac{1}{r} \frac{\partial}{\partial r}\left(r \frac{\partial n}{\partial r}\right)\right)
\end{aligned}
$$


With the boundary conditions are of the form:

$v=0, T=T_{w}(x), C=C_{\infty}(x), n=n_{\infty}(x) \quad$ at $\quad r=r_{0}$

$u \rightarrow U(x), T \rightarrow T_{\infty}, C \rightarrow C_{\infty}, n \rightarrow n_{\infty}$ at $r=\infty$

Following Mahmood and Merkin [42], assuming in this paper the dimensionless quantities:

$$
\begin{aligned}
& \eta=\frac{r^{2}-r_{0}{ }^{2}}{2 r_{0} L}\left(P e^{\frac{1}{2}}+R a^{\frac{1}{2}}\right) \psi=\alpha r_{0}\left(P e^{\frac{1}{2}}+R a^{\frac{1}{2}}\right) \frac{x}{L} f(\eta) \\
& T=T_{\infty}+\frac{a x^{m+1} \nabla T}{L} \theta(\eta), C=C_{\infty}+\frac{b x^{m+1} \nabla C}{L} \varphi(\eta), n=n_{\infty}+\frac{c x^{m+1} \nabla n}{L} \chi(\eta)
\end{aligned}
$$

The continuity equation is satisfied by stream function such that $u=\frac{1}{r} \frac{\partial \psi}{\partial r} v=-\frac{1}{r} \frac{\partial \psi}{\partial x}$. Using Eq. (8) and (9) in Eq. (1) to (7) leads to the following coupled differential equations:

$$
\begin{aligned}
& f^{\prime \prime}=(1-\lambda)^{2}\left[\theta^{\prime}+N_{1} \phi^{\prime}+N_{2} \chi^{\prime}\right] \operatorname{Cos} \omega \\
& (1+\gamma \eta) \theta^{\prime \prime}+\gamma \theta^{\prime}+f \theta^{\prime}-(m+1) f^{\prime} \theta=0 \\
& (1+\gamma \eta) \varphi^{\prime \prime}+\gamma \varphi^{\prime}+\text { Le.f } f \varphi^{\prime}-(m+1) L e \cdot f^{\prime} \phi=0 \\
& (1+\gamma \eta) \chi^{\prime \prime}+\gamma \chi^{\prime}+L b \cdot f \chi^{\prime}-L b \cdot(m+1) f^{\prime} \chi-P e\left((1+\gamma \eta) \phi^{\prime} \chi^{\prime}+(\chi+A)\left(\gamma \varphi^{\prime}+(1+\gamma \eta) \phi^{\prime \prime}\right)\right)=0
\end{aligned}
$$

The transformed boundary conditions become:

$$
\begin{aligned}
& \eta=0, f=0, \theta=1, \varphi=1, \chi=1 \text { and } \\
& \eta \rightarrow \infty, f^{\prime} \rightarrow \lambda^{2}, \theta \rightarrow 0, \varphi \rightarrow 0, \chi \rightarrow 0
\end{aligned}
$$

Where $\lambda=\left(1+\frac{R a^{\frac{1}{2}}}{P e^{\frac{1}{2}}}\right)^{-1}, R a=\frac{g \beta k L a x^{m} \nabla T}{v \alpha}, P e=\frac{u_{\infty} L}{\alpha}, \gamma=\frac{2 L}{r_{0}\left(P e^{\frac{1}{2}}+R a^{\frac{1}{2}}\right)}, N_{1}=\frac{\beta_{c} \nabla C}{\beta_{T} \nabla T} N_{2}=\frac{\beta_{n} \nabla n}{\beta_{T} \nabla T}$ $L e=\frac{\alpha}{D_{m}}, L b=\frac{\alpha}{D_{n}}, P e=\frac{b W_{c}}{D_{n}}, A=\frac{n_{\infty}}{n_{w}-n_{\infty}}$ 


\subsection{Numerical Procedure}

Using similarity transformations, the governing partial differential equations were converted into ordinary differential equations and then numerically solved by using by MATLAB bvp4c solver. In this method, using the different initial guesses of $f, f^{\prime}, \theta, \theta^{\prime}, \phi, \phi^{\prime}, \chi, \chi^{\prime}$ we can able to find the first and second solution. In the context of bvp4c function described above we need to transform the governing Eq. (10) - (13) into first order differential equation letting $\eta=x$ and

$y_{1}=f, y_{2}=f^{\prime}, y_{3}=\theta, y_{4}=\theta^{\prime}, y_{5}=\phi, y_{6}=\phi^{\prime}, y_{7}=\chi, y_{8}=\chi^{\prime}$

The corresponding first order differential equations are

$$
\begin{aligned}
& \frac{d y_{1}}{d x}=f^{\prime}=y_{2}, \frac{d y_{2}}{d x}=f^{\prime \prime}=(1-\lambda)^{2}\left[y_{4}+N_{1} y_{6}+N_{2} y_{8}\right] \\
& \frac{d y_{3}}{d x}=\theta^{\prime}=y_{2}, \frac{d y_{4}}{d x}=\theta^{\prime \prime}=\frac{(m+1) y_{2} y_{3}-\gamma y_{4}-y_{1} y_{4}}{(1+\gamma \eta)} \\
& \frac{d y_{5}}{d x}=\varphi^{\prime}=y_{6}, \frac{d y_{6}}{d x}=\varphi^{\prime \prime}=\frac{(m+1) L e y_{2} y_{5}-\gamma y_{6}-L e y_{1} y_{6}}{(1+\gamma \eta)} \\
& \frac{d y_{7}}{d x}=\chi^{\prime}=y_{8},=\frac{d y_{8}}{d x}=\chi^{\prime \prime}=\frac{L e y_{1} y_{6}+L e(m+1) y_{2} y_{5}}{\left(1+\gamma \eta y_{2} y_{7}-\gamma y_{8}-L b y_{1} y_{8}+P e\left((1+\gamma \eta) y_{6} y_{8}+\left(y_{7}+A\right)\left(\gamma y_{6}+(1+\gamma \eta)\left(-\gamma y_{6}-\right.\right.\right.\right.}
\end{aligned}
$$

The boundary conditions (14) and (15) become considering ya be the left boundary, $y b$ be the right boundary

$$
\begin{aligned}
& y a(1)=0, y(b)-\lambda^{2}=0 \\
& y a(3)-1=0, y b(3)=0 \\
& y a(5)-1=0, y b(5)=0 \\
& y a(7)-1=0, y b(7)=0
\end{aligned}
$$

For the validation of the results the differential equations are again solved numerically using Maple 14.0 with the help of dsolve command where asymptotic boundary conditions (14) and (15) are replaced by using a value of 5 for the similarity variable $\eta_{\max }=5$. The obtaining results for both cases as shown in Table 1 shows good agreement and exactness of numerical calculations.

Table 1

Effect of Mixed Convection parameter $\lambda$ on $-\theta^{\prime}(0)$ when

$$
N_{1}=0.5, N_{2}=0.6, m=0.2, \gamma=0.1, \omega=\frac{\pi}{4}, L b=0.0, L e=0.0, P e=0.0, A=0.0
$$

\begin{tabular}{lll}
\hline$\lambda$ & $-\theta^{\prime}(0)$ (Matlab Bvp4c) & $-\theta^{\prime}(0)$ (Maple 14.0) \\
\hline & First Solution & First Solution \\
0.0 & 1.4543 & 1.4550 \\
0.3 & 1.0922 & 1.0929 \\
0.5 & 0.9937 & 0.9941 \\
0.8 & 1.1238 & 1.1239 \\
1.0 & 1.3520 & 1.3520 \\
\hline
\end{tabular}


For further confirmation, the present results for the special case are validated against the results of investigations by Chamkha and Khaled [43], Gorla et al., [44] and Nima et al., [45] shown in Table 2.

\section{Table 2}

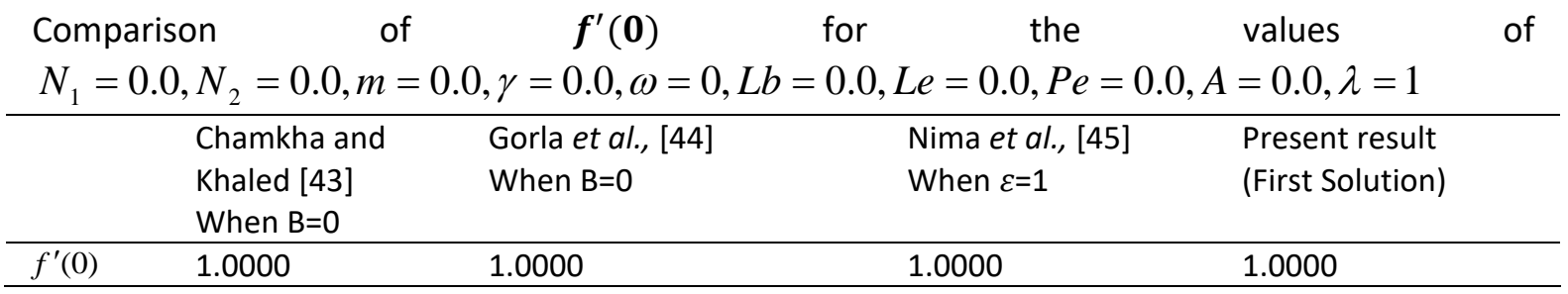

A stability analysis described by Sparrow et al., [46], Weidman et al., [47] and very recently Postelnicu and Pop [48] reveal that upper branch (first solution) solutions are stable and physically accomplishable, while lower branch solutions (second solution) are unstable and therefore, not physically accomplishable. As their researches are in similar physical situation, so we will not repeat that analysis here in this paper. All the figures show that dual solutions for all values of $\lambda_{c} \leq \lambda$ where $\lambda_{c}$ is the critical value.

\section{Results}

\subsection{Analysis of Velocity Profile}

In Figure 2(a), dual solution for velocity profile $f^{\prime}(\eta)$ against $\eta$ for several values of mixed convection parameter $\lambda$ is shown. Mixed convection parameter $\lambda$ covers entire regime of mixed convection from pure free convection (when $\lambda=0$ ) to pure forced convection (when $\lambda=1$ ). Velocity profile increases with the growing values of curvature parameter $\gamma$ in Figure 2(b). Velocity profile decreases with the increases of curvature parameter. For the higher values of curvature parameter, the radius of cylinder decreases. Less contact within surface area produces less amount of resistance towards fluid particles, as a result the velocity profile shows a stimulant value. It is noticed in Figure 2(a) and Figure 2(b) that first solution is stable as the velocity profile went into positive range and the second solution is unstable as the velocity profile went out negative. The velocity flow profiles provide the existence of the dual solution when $\lambda>\lambda_{C}$.

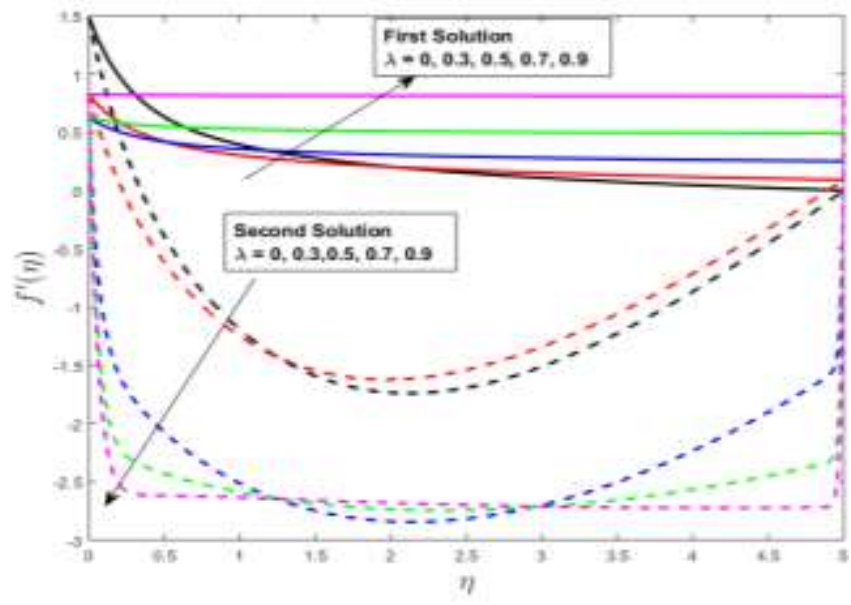

(a)

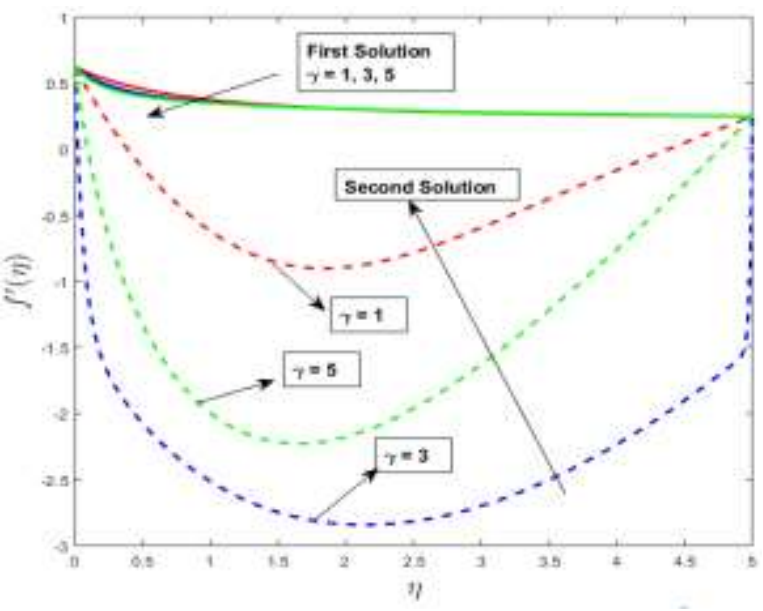

(b)

Fig. 2. Velocity profile with different values of (a) Mixed Convection parameter $\lambda$ (b) Curvature parameter $\gamma$ 


\subsection{Heat Transfer Rate and Temperature Profile}

Variation of Nusselt number with $\lambda$ for different values of $\gamma$ is shown in Figure 3(a). So, it is seen that dual solution exists for the temperature profile $\lambda>\lambda_{c}$ where $\lambda_{c}=0.31,0.28,0.24$ when $\gamma=$ $0.3,0.4,0.5$ respectively. Nusselt number decreases from free convection to mixed convection regime, after that it gradually increases to forced convective regime with the growing values of curvature parameter $\boldsymbol{\lambda}$. It is also noticed that dual solution exists in free convective regime. We know that increase in curvature parameter cause decrease in radius of curvature because of which velocity of fluid particles enhances. As a result, average kinetic energy increase which causes an increment in temperature profile. So, Figure 3(a) and Figure 4(a) illustrate heat transfer rate and temperature profile increase for the higher values of $\lambda$ for the first solution but opposite phenomena are observed for second solution.

In Figure 3(b) variation of heat transfer rate with $\lambda$ different values of power law exponent $\mathrm{m}$ is shown where heat transfer rate increases with the increasing values of $m$. Dual solution exists for $\lambda$ $>\lambda_{c}$ where $\lambda_{c}=0.20,0.25,0.28$ when $m=0.6,0.8,1.0$ respectively. The temperature profile as well as the thermal boundary layer thickness reduces in Figure 4(b) with the increase of power law index, $m$ because fluid velocity increases for low viscosity which lessens fluid temperature. In summary to increase the value of $m$ is to increase the heat transfer rate and to decrease the thermal boundary layer. So, it is clearly stated that second solutions observed in Figure 3(b) and Figure 4(b) are not physically realizable.

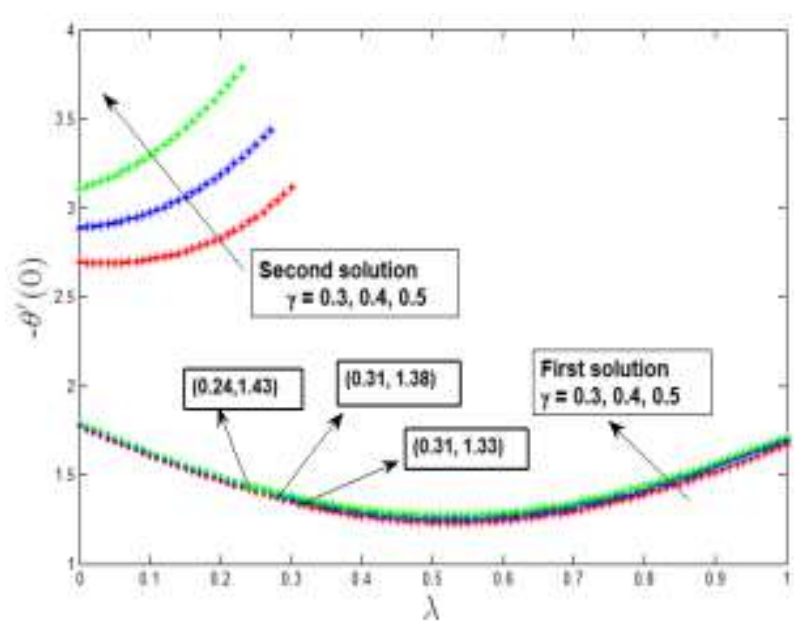

(a)

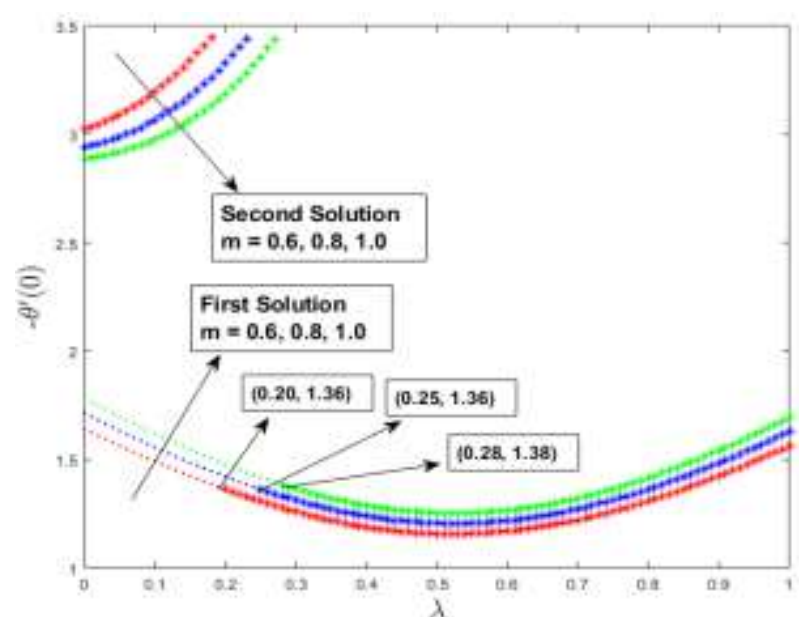

(b)

Fig. 3. Heat transfer rate with mixed convection parameter $\lambda$ for different values of (a) Curvature parameter $\mathrm{y}$ (b) Power law exponent $\mathrm{m}$ 


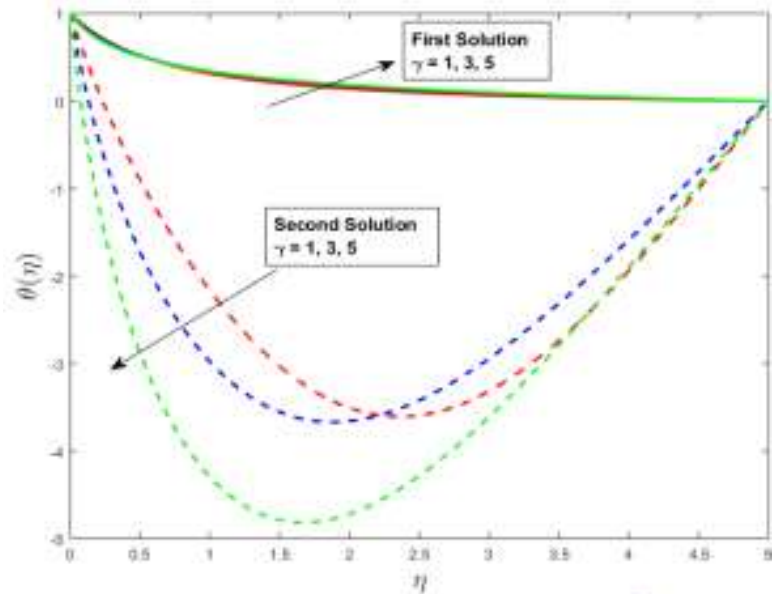

(a)

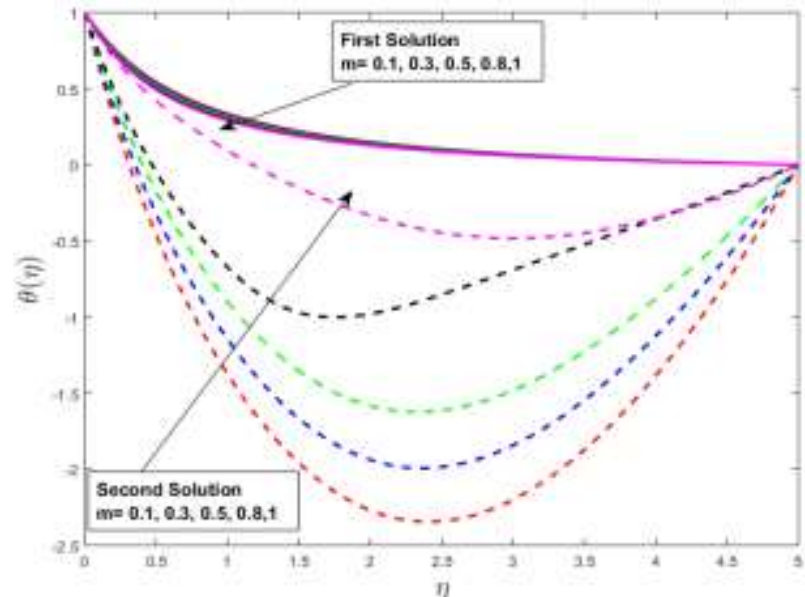

(b)

Fig. 4. Temperature profile with different values of (a) Curvature parameter $\gamma$ (b) Power law exponent $m$

\subsection{Mass Transfer Rate and Concentration Profile}

Variation of Sherwood number with $\lambda$ for different values of $\gamma$ is shown in Figure 5(a) the dual solution is observed for the concentration profile $\lambda>\lambda_{c}$ where $\lambda_{c}=0.10,0.17$ when $\gamma=0.2,0.5$ respectively. At this point $\lambda_{c}$ unique solution exists. It is also observed that the Sherwood number decreases in free convection regime both for first and second solution, after that it increases from mixed convective to forced convective regime with the increasing values of $\lambda$ and $\gamma$ for the first solution. In Figure $6(\mathrm{a})$ existence of dual solution of concentration profile when $\lambda>\lambda_{c}$ for the values $\gamma=0.1,0.5,1.0$ is shown. Boundary layer thickness of concentration profile increases with the increasing values of $\gamma$ for the first solution and decreases for the second solution.

Effect of power law exponent $m$ on Mass transfer rate with the variation of $\boldsymbol{\lambda}$ is observed in Figure 5 (b) provides the existence of the dual solution for the variation of power law exponent $m$ when $\lambda>$ $\lambda_{C}$. For $\mathrm{m}=0.6,0.8,1.0$ we get the critical points $\lambda_{C}=0.03,0.07,0.10$ where unique solutions exist. For the first solution mass transfer rate increases with $\mathrm{m}$. Decreasing of boundary layer thickness of concentration profile is observed for Lewis parameter Le in Figure 6(b). Unstable phenomena is seen for the second solutions.

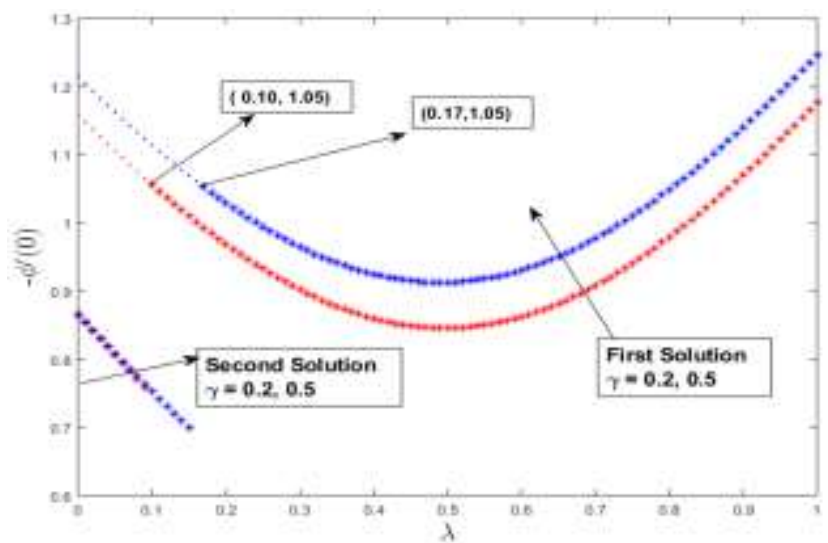

(a)

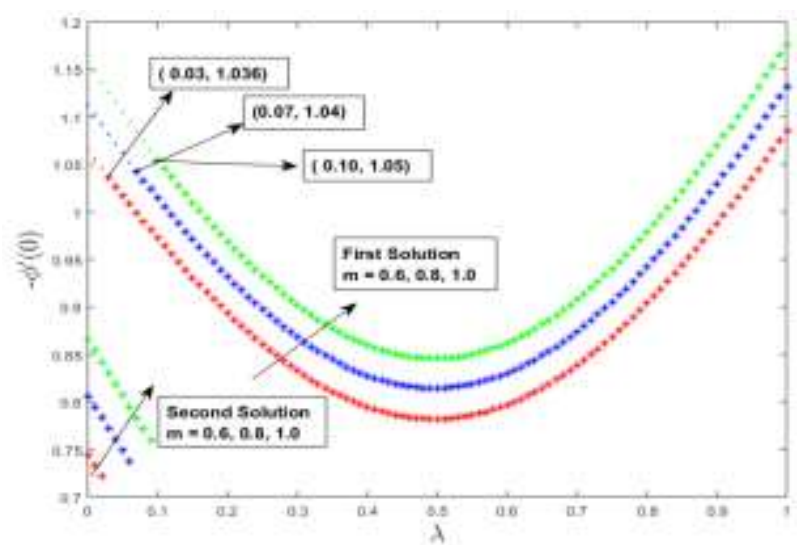

(b)

Fig. 5. Mass transfer rate with mixed convection parameter $\lambda$ for different values of (a) Curvature parameter $\gamma$ (b) Power law exponent $m$ 


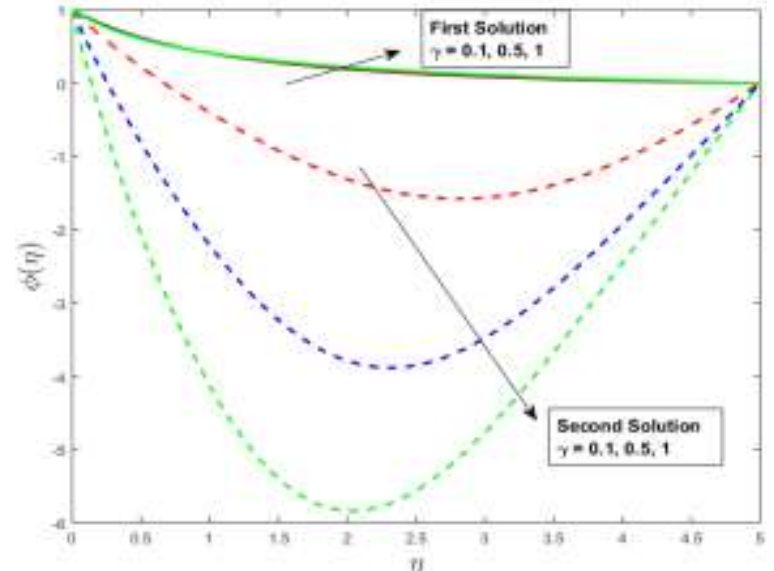

(a)

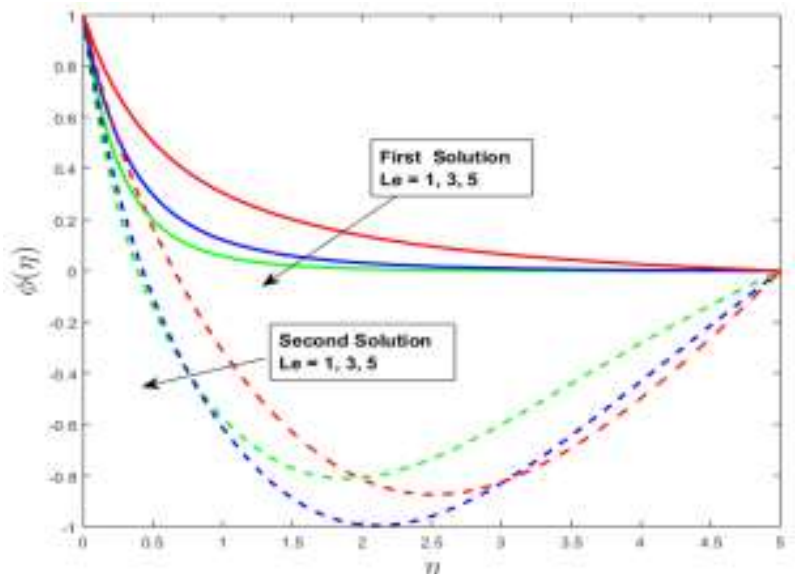

(b)

Fig. 6. Concentration profile with different values of (a) Curvature parameter $\psi$ (b) Lewis Parameter Le

\subsection{Motile Microorganism Transfer Rate and Microorganism Profiles}

Dual solutions for Motile Microorganism transfer rate with mixed convection parameter $\lambda$ are observed in Figure 7(a) and Figure 7(b). The dual solutions are observed for the microorganism profile $\lambda>\lambda_{c}$ where $\lambda_{c}=0.28,0.27,0.26$ when $\gamma=0.1,0.2,0.3$ and also when $m=0.8,0.9,1.0, \lambda_{c}=$ $0.25,0.26,0.27$. As motile microorganism density is higher than the liquid and they normally swim in upward way for the exterior of the cylinder wall, so growing values of curvature parameter increases motile microorganism transfer rate and decreases boundary layer thickness of microorganism profile which are presented in Figure 7(a) and Figure 8(a) for the stable solutions. Motile microorganism transfer rate increases also for power law exponent $\mathrm{m}$. which is illustrated in Figure 7(b).

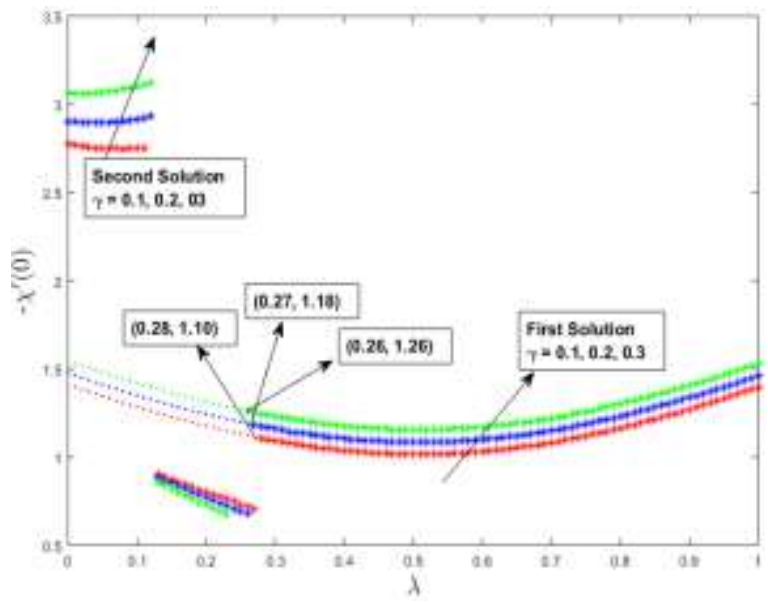

(a)

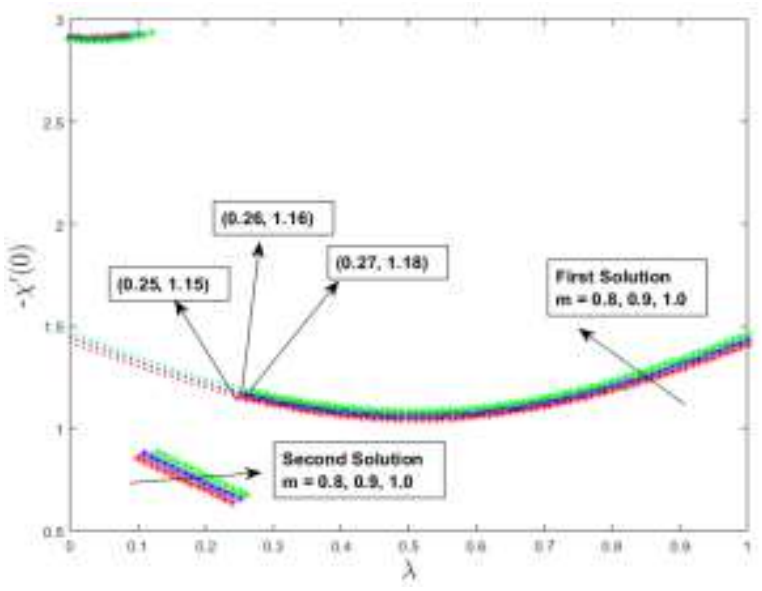

(b)

Fig. 7. Microorganism transfer rate with mixed convection parameter $\lambda$ for different values of (a) Curvature Parameter $y$ (b) Power law exponent $m$

In Figure $8(\mathrm{~b})$ and Figure $8(\mathrm{c})$ existence of dual solution of microorganism profile when $\lambda>\lambda_{c}$ for the values $\mathrm{Lb}=0.1,0.5,0.8$ and $\mathrm{Pe}=0.1,0.3,0.5$ is shown. In both figure we can see boundary layer thickness of microorganism profile decreases with the growing values of $L b$ and Pe. Increasing the bioconvection Lewis number Lb causes decrement of motile microorganism diffusivity, which results in microorganism's concentration reduction. On the other hand, increasing bioconvection peclet number rises the mobility of fluid causes quantity of motile microorganism's thickness reduces, hence microorganism's diffusivity and their concentration decreases. It is also noticed that first 
solution is stable as the Microorganism profile went into positive range and the second solution is unstable as the profile went out negative.

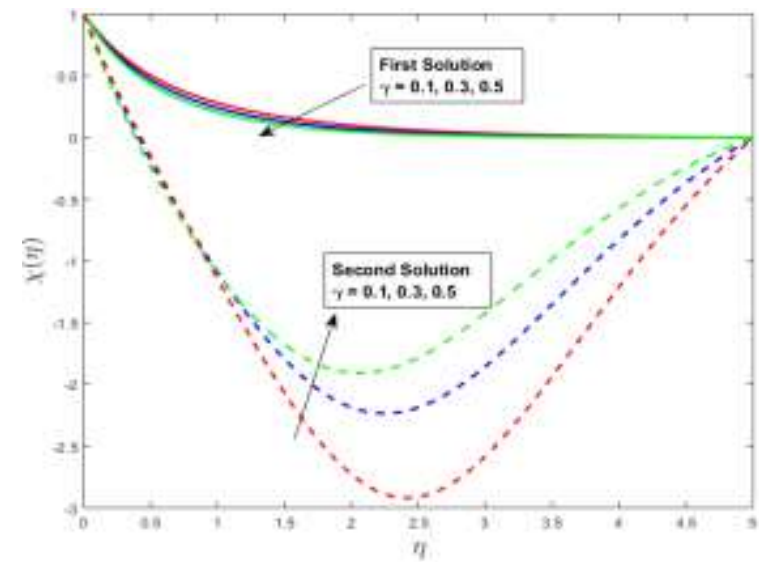

(a)

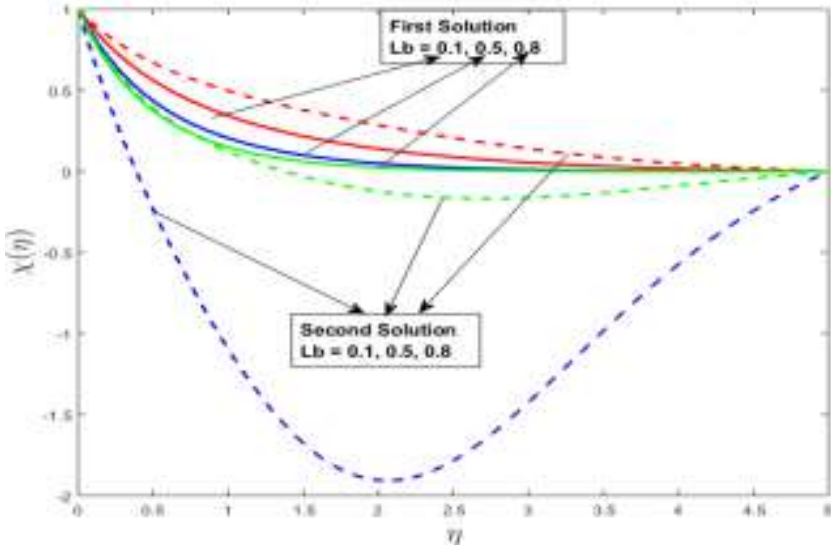

(b)

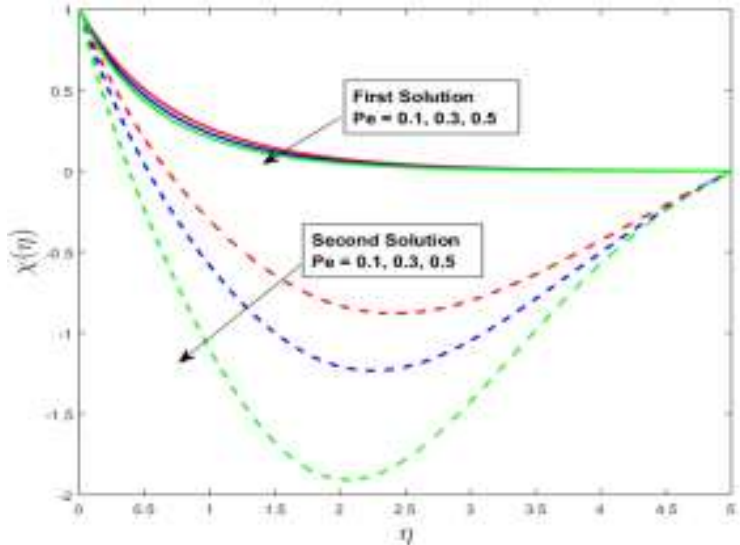

(c)

Fig. 8. Microorganism profile with different values of (a) Curvature parameter $\gamma$ (b) Bioconvection Lewis Number (c) Bioconvection Peclet number Pe

\section{Conclusion}

The steady mixed convection boundary layer flow with gyrotactic microorganism past an inclined cylinder is analyzed. Results indicate that multiple solutions exist in free convection dominated regime. Critical point separated upper branch and lower branch solutions. Stable solutions were indicated by upper branch and unstable solutions were also indicated by lower branch. The consequences of various flow influencing parameters have been thoroughly discussed in detail. The key reviews are condensed as follows

i. The variation of nusselt number indicates that dual solution exists for temperature profile $\lambda>$ $\lambda_{c}$ where the critical value $\lambda_{c}=0.31,0.28,0.24$ for the curvature parameter $\gamma=0.3,0.4,0.5$ and the critical value $\lambda_{c}=0.20,0.25,0.28$ for the exponent $m=0.6,0.8,1.0$.

ii. The variation of Sherwood number shows the existence of dual solution in concentration profile when $\lambda>\lambda_{c}=0.10,0.17$. for $\gamma=0.2,0.5$ and $\lambda_{c}=0.03,0.07,0.10$ for the $m=$ $0.6,0.8,1.0$ respectively.

iii. The variations of density number of microorganisms show the dual solution of Microorganism profile arise when $\lambda>\lambda_{c}=0.25,0.26,0.27$ for $\gamma=0.1,0.2,0.3$ and $\lambda_{c}=0.25,0.26,0.27$ for the $m=0.8,0.9,1.0$ respectively. 


\section{Acknowledgement}

This research was not funded by any grant.

\section{References}

[1] Bachok, Norfifah, Anuar Ishak, and loan Pop. "Mixed convection boundary layer flow over a moving vertical flat plate in an external fluid flow with viscous dissipation effect." PLoS ONE 8, no. 4 (2013): e60766. https://doi.org/10.1371/journal.pone.0060766

[2] Rahman, M. M., J. H. Merkin, and I. Pop. "Mixed convection boundary-layer flow past a vertical flat plate with a convective boundary condition." Acta Mechanica 226, no. 8 (2015): 2441-2460. https://doi.org/10.1007/s00707015-1334-2

[3] Rana, Puneet, R. Bhargava, and O. Anwar Bég. "Numerical solution for mixed convection boundary layer flow of a nanofluid along an inclined plate embedded in a porous medium." Computers \& Mathematics with Applications 64, no. 9 (2012): 2816-2832. https://doi.org/10.1016/j.camwa.2012.04.014

[4] Mondal, Hiranmoy, Poulomi De, Sicelo Goqo, and Precious Sibanda. "A Numerical Study of Nanofluid Flow Over a Porous Vertical Plate with Internal Heat Generation and Nonlinear Thermal Radiation." Journal of Porous Media 23, no. 6 (2020): 517-529. https://doi.org/10.1615/JPorMedia.2020026624

[5] Abu-Hamdeh, Nidal H., Hakan F. Oztop, and Khalid A. Alnefaie. "A computational study on mixed convection in a porous media filled and partially heated lid-driven cavity with an open side." Alexandria Engineering Journal 59, no. 3 (2020): 1735-1750. https://doi.org/10.1016/i.aej.2020.04.039

[6] Maleque, Kh. Abdul. "Similarity Requirements for Mixed Convective Boundary Layer Flow over Vertical Curvilinear Porous Surfaces with Heat Generation/Absorption." International Journal of Aerospace Engineering 2020 (2020). https://doi.org/10.1155/2020/7486971

[7] Gangadhar, K., R. Edukondala Nayak, and M. Venkata Subba Rao. "Buoyancy effect on mixed convection boundary layer flow of Casson fluid over a non linear stretched sheet using the spectral relaxation method." International Journal of Ambient Energy (2020): 1-9. https://doi.org/10.1080/01430750.2020.1722963

[8] Zhao, Jinhu. "Finite volume method for mixed convection boundary layer flow of viscoelastic fluid with spatial fractional derivatives over a flat plate." Computational and Applied Mathematics 40, no. 1 (2021): 1-17. https://doi.org/10.1007/s40314-020-01394-2

[9] Bakar, Norhaliza Abu, and Rozaini Roslan. "Mixed Convection in a Lid-Driven Horizontal Cavity in the Presence of Internal Heat Generation or Absorption." Journal of Advanced Research in Numerical Heat Transfer 3, no. 1 (2020): 1-11.

[10] Popiel, Czeslaw O. "Free convection heat transfer from vertical slender cylinders: a review." Heat Transfer Engineering 29, no. 6 (2008): 521-536. https://doi.org/10.1080/01457630801891557

[11] Loganathan, P., and B. Eswari. "Natural convective flow over moving vertical cylinder with temperature oscillations in the presence of porous medium." Global Journal of Pure and Applied Mathematics 13, no. 2 (2017): 839-855.

[12] Sewucipto, Sanjaya, and Triyogi Yuwono. "The Influence of Upstream Installation of D-53 Type Cylinder on the Performance of Savonius Turbine." Journal of Advanced Research in Experimental Fluid Mechanics and Heat Transfer 3, no. 1 (2021): 36-47.

[13] Rashidi, M. M., O. Anwar Bég, and N. Rahimzadeh. "A generalized differential transform method for combined free and forced convection flow about inclined surfaces in porous media." Chemical Engineering Communications 199, no. 2 (2012): 257-282. https://doi.org/10.1080/00986445.2011.586757

[14] Dhanai, Ruchika, Puneet Rana, and Lokendra Kumar. "MHD mixed convection nanofluid flow and heat transfer over an inclined cylinder due to velocity and thermal slip effects: Buongiorno's model." Powder Technology 288 (2016): 140-150. https://doi.org/10.1016/j.powtec.2015.11.004

[15] Rihan, Yasser A. "Mixed Convection Heat Transfer from a Short Vertical Cylinder Placed in a Cross Flow." ERJ Engineering Research Journal 43, no. 3 (2020): 195-197. https://doi.org/10.21608/erjm.2020.95137

[16] Mkhatshwa, M. P., S. S. Motsa, M. S. Ayano, and P. Sibanda. "MHD mixed convective nanofluid flow about a vertical slender cylinder using overlapping multi-domain spectral collocation approach." Case Studies in Thermal Engineering 18 (2020): 100598. https://doi.org/10.1016/i.csite.2020.100598

[17] Ali, Mohamed E. "On thermal boundary layer on a power-law stretched surface with suction or injection." International Journal of Heat and Fluid Flow 16, no. 4 (1995): 280-290. https://doi.org/10.1016/0142727X(95)00001-7

[18] Ferdows, M., M. G. Murtaza, and M. D. Shamshuddin. "Effect of internal heat generation on free convective powerlaw variable temperature past a vertical plate considering exponential variable viscosity and thermal conductivity." Journal of the Egyptian Mathematical Society 27, no. 56 (2019): 1-11. https://doi.org/10.1186/s42787-019-0062-5 
[19] Muñoz-Cobo, José L., José M. Corberán, and Sergio Chiva. "Explicit formulas for laminar natural convection heat transfer along vertical cylinders with power-law wall temperature distribution." Heat and Mass Transfer 39, no. 3 (2003): 215-222. https://doi.org/10.1007/s00231-002-0310-2

[20] Kim, Minsung, Jeong Hoon Doo, Yong Gap Park, Hyun Sik Yoon, and Man Yeong Ha. "Natural convection in a square enclosure with a circular cylinder according to the bottom wall temperature variation." Journal of Mechanical Science and Technology 28, no. 12 (2014): 5013-5025. https://doi.org/10.1007/s12206-014-1123-1

[21] Ghorai, S., and N. A. Hill. "Development and stability of gyrotactic plumes in bioconvection." Journal of Fluid Mechanics 400 (1999): 1-31. https://doi.org/10.1017/S0022112099006473

[22] Ghorai, S., and N. A. Hill. "Periodic arrays of gyrotactic plumes in bioconvection." Physics of Fluids 12, no. 1 (2000): 5-22. https://doi.org/10.1063/1.870249

[23] Mahdy, A. "Gyrotactic microorganisms mixed convection nanofluid flow along an isothermal vertical wedge in porous media." International Journal of Aerospace and Mechanical Engineering 11, no. 4 (2017): 840-850.

[24] Khan, Noor Saeed, Taza Gul, Muhammad Altaf Khan, Ebenezer Bonyah, and Saeed Islam. "Mixed convection in gravity-driven thin film non-Newtonian nanofluids flow with gyrotactic microorganisms." Results in Physics 7 (2017): 4033-4049. https://doi.org/10.1016/j.rinp.2017.10.017

[25] Khan, Waqar A., A. M. Rashad, M. M. M. Abdou, and I. Tlili. "Natural bioconvection flow of a nanofluid containing gyrotactic microorganisms about a truncated cone." European Journal of Mechanics-B/Fluids 75 (2019): $133-142$. https://doi.org/10.1016/j.euromechflu.2019.01.002

[26] Saleem, S., Hunza Rafiq, A. Al-Qahtani, Mohamed Abd El-Aziz, M. Y. Malik, and I. L. Animasaun. "Magneto Jeffrey nanofluid bioconvection over a rotating vertical cone due to gyrotactic microorganism." Mathematical Problems in Engineering 2019 (2019). https://doi.org/10.1155/2019/3478037

[27] Sudhagar, Palani, Peri K. Kameswaran, and B. Rushi Kumar. "Gyrotactic Microorganism Effects on Mixed Convective Nanofluid Flow Past a Vertical Cylinder." Journal of Thermal Science and Engineering Applications 11, no. 4 (2019): 041018. https://doi.org/10.1115/1.4044185

[28] Mallikarjuna, B., A. M. Rashad, Ali Chamkha, and M. M. M. Abdou. "Mixed bioconvection flow of a nanofluid containing gyrotactic microorganisms past a vertical slender cylinder." Frontiers in Heat and Mass Transfer (FHMT) 10 (2018). https://doi.org/10.5098/hmt.10.21

[29] Rashad, A. M., and Hossam A. Nabwey. "Gyrotactic mixed bioconvection flow of a nanofluid past a circular cylinder with convective boundary condition." Journal of the Taiwan Institute of Chemical Engineers 99 (2019): 9-17. https://doi.org/10.1016/i.jtice.2019.02.035

[30] Mahdy, A., and Hossam A. Nabwey. "Microorganisms time-mixed convection nanofluid flow by the stagnation domain of an impulsively rotating sphere due to Newtonian heating." Results in Physics 19 (2020): 103347. https://doi.org/10.1016/j.rinp.2020.103347

[31] Subhashini, S. V., and R. Sumathi. "Dual solutions of a mixed convection flow of nanofluids over a moving vertical plate." International Journal of Heat and Mass Transfer 71 (2014): 117-124. https://doi.org/10.1016/j.ijheatmasstransfer.2013.12.034

[32] Ridha, A., and Marie Curie. "Aiding flows non-unique similarity solutions of mixed-convection boundary-layer equations." Zeitschrift für angewandte Mathematik und Physik ZAMP 47, no. 3 (1996): 341-352. https://doi.org/10.1007/BF00916642

[33] Subhashini, S. V., R. Sumathi, and I. Pop. "Dual solutions in a double-diffusive MHD mixed convection flow adjacent to a vertical plate with prescribed surface temperature." International Journal of Heat and Mass Transfer 56, no. 12 (2013): 724-731. https://doi.org/10.1016/i.ijheatmasstransfer.2012.08.065

[34] Ingham, D. B. "Singular and non-unique solutions of the boundary-layer equations for the flow due to free convection near a continuously moving vertical plate." Zeitschrift für angewandte Mathematik und Physik ZAMP 37, no. 4 (1986): 559-572. https://doi.org/10.1007/BF00945430

[35] Merkin, J. H. "On dual solutions occurring in mixed convection in a porous medium." Journal of Engineering Mathematics 20, no. 2 (1986): 171-179. https://doi.org/10.1007/BF00042775

[36] Rostami, Mohammadreza Nademi, Saeed Dinarvand, and loan Pop. "Dual solutions for mixed convective stagnation-point flow of an aqueous silica-alumina hybrid nanofluid." Chinese Journal of Physics 56, no. 5 (2018): 2465-2478. https://doi.org/10.1016/i.cjph.2018.06.013

[37] Salleh, Siti Nur Alwani, Norfifah Bachok, Norihan Md Arifin, Fadzilah Md Ali, and loan Pop. "Stability analysis of mixed convection flow towards a moving thin needle in nanofluid." Applied Sciences 8, no. 6 (2018): 842. https://doi.org/10.3390/app8060842

[38] Khan, M. Riaz, Kejia Pan, Arif Ullah Khan, and S. Nadeem. "Dual solutions for mixed convection flow of SiO2Al203/water hybrid nanofluid near the stagnation point over a curved surface." Physica A: Statistical Mechanics and its Applications 547 (2020): 123959. https://doi.org/10.1016/i.physa.2019.123959 
[39] Grosan, T., and I. Pop. "Axisymmetric mixed convection boundary layer flow past a vertical cylinder in a nanofluid." International Journal of Heat and Mass Transfer 54, no. 15-16 (2011): 3139-3145. https://doi.org/10.1016/j.ijheatmasstransfer.2011.04.018

[40] Shu, Jian-Jun, Qi-Wen Wang, and Ioan Pop. "Dual solutions for opposing mixed convection in porous media." Journal of Heat Transfer 139, no. 10 (2017): 102501. https://doi.org/10.1115/1.4036727

[41] Ferdows, M., Amran Hossan, M. Z. I. Bangalee, Shuyu Sun, and Faris Alzahrani. "Stability theory of nano-fluid over an exponentially stretching cylindrical surface containing microorganisms." Scientific Reports 10, no. 1 (2020): 118. https://doi.org/10.1038/s41598-020-72545-y

[42] Mahmood, T., and J. H. Merkin. "Mixed convection on a vertical circular cylinder." Zeitschrift für angewandte Mathematik und Physik ZAMP 39, no. 2 (1988): 186-203. https://doi.org/10.1007/BF00945765

[43] Chamkha, A. J., and A-RA Khaled. "Hydromagnetic simultaneous heat and mass transfer by mixed convection from a vertical plate embedded in a stratified porous medium with thermal dispersion effects." Heat and Mass Transfer 36 (2000): 63-70. https://doi.org/10.1007/s002310050365

[44] Gorla, Rama Subba Reddy, A. Y. Bakier, and L. Byrd. "Effects of thermal dispersion and stratification on combined convection on a vertical surface embedded in a porous medium." Transport in Porous Media 25, no. 3 (1996): $275-$ 282. https://doi.org/10.1007/BF00140984

[45] Nima, Nayema Islam, M. Ferdows, and M. Moghimi Ardekani. "Effects of Cross diffusion and radiation on magneto mixed convective stagnation flow from a vertical surface in porous media with Gyrotactic Microorganisms: similarity and numerical analysis." Special Topics \& Reviews in Porous Media: An International Journal 11, no. 3 (2020). https://doi.org/10.1615/SpecialTopicsRevPorousMedia.2020030455

[46] Sparrow, Ephraim M., S. V. Patankar, and S. Ramadhyani. "Analysis of Melting in the Presence of Natural Convection in the Melt Region." Trans. ASME Journal of Heat Transfer 99, no. 4 (1977): 520-526. https://doi.org/10.1115/1.3450736

[47] Weidman, P. D., D. G. Kubitschek, and A. M. J. Davis. "The effect of transpiration on self-similar boundary layer flow over moving surfaces." International Journal of Engineering Science 44, no. 11-12 (2006): $730-737$. https://doi.org/10.1016/j.ijengsci.2006.04.005

[48] Postelnicu, A., and Ioan Pop. "Falkner-Skan boundary layer flow of a power-law fluid past a stretching wedge." Applied Mathematics and Computation 217, no. 9 (2011): 4359-4368. https://doi.org/10.1016/i.amc.2010.09.037 